\section{Óxido nítrico exhalado, asma y atopia: análisis en un panel de niños en Santiago, Chile}

\author{
DANIELLA VIDAL ${ }^{1, \mathrm{a}}$, KARLA YOHANNESSEN $^{2, \mathrm{~b}}$, \\ LAURA PRIETO ${ }^{2, \mathrm{c}}$, CARLOS UBILLA $^{1}$, PABLO A. RUIZ ${ }^{2, \mathrm{~d}}$
}

\section{Association of exhaled nitric oxide with asthma and atopy among children living in Santiago, Chile}

Background: Chronic airway inflammation is a central process in asthma. Measurement of exhaled nitric oxide (eNO) is a non-invasive biomarker of eosinophilic airway inflammation. Aim: To measure eNO levels in a population of asthmatic and non-asthmatic children and to evaluate their relationship with asthma and atopy. Material and Methods: We studied 143 asthmatic and non-asthmatic children aged 6 to 14 years attended a hospital and primary health service. Participants were tested for allergies and followed during the winter months of 2010 and 2011. They were visited regularly at their homes and eNO levels were measured on each visit using a handheld equipment. Mean eNO distribution were compared by the presence of asthma or atopy using t-test and regression models. Results: No significant differences for mean eNO levels were detected, according to presence of asthma or atopy, by any of the statistical methods used. Regression models showed significant effects for age but not for sex. Conclusions: There were no differences in eNO levels in the studied children by the presence of asthma or atopy.

(Rev Med Chile 2013; 141: 743-750).

Key words: Asthma; Child; Chile; Hypersensitivity; Nitric oxide.

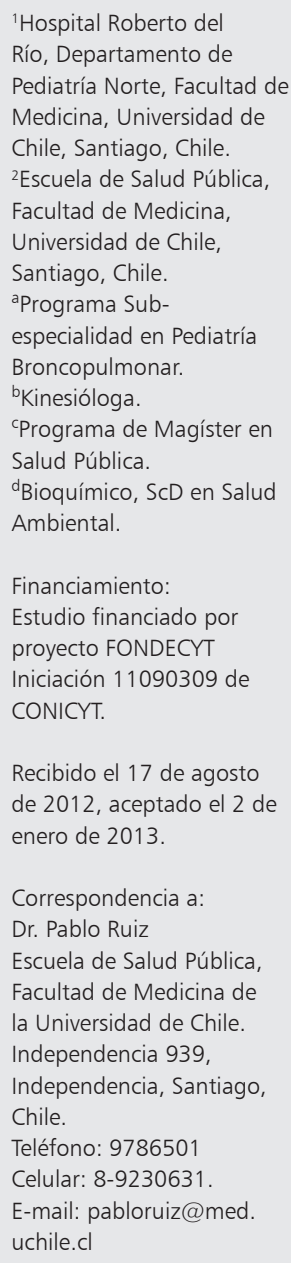

U na de las características distintivas del asma bronquial es la inflamación crónica de la vía aérea ${ }^{1,2}$. Esta inflamación es de difícil evaluación y no está incorporada en forma rutinaria ni en el proceso habitual de diagnóstico, ni como guía de tratamiento. La inflamación de la vía aérea se puede medir por varios métodos, algunos de ellos invasivos, como biopsia de mucosa bronquial y lavado broncoalveolar. En los últimos años existe un gran desarrollo en la evaluación no invasiva de la inflamación de la vía aérea, siendo la medición del óxido nítrico exhalado $(\mathrm{ONe})$ el método más estudiado. El valor de la medición de ONe reside en su capacidad de medir la inflamación de las vías aéreas, en especial el fenotipo de asma con inflamación de tipo eosinofílica.
Estudios muestran que los niveles de $\mathrm{ONe}$ se correlacionan con los recuentos de eosinófilos en muestras de esputo inducido, lavado broncoalveolar y eosinófilos en biopsia bronquial ${ }^{3-5}$.

Actualmente, se ha estandarizado la medición de ONe para uso clínico ${ }^{6}$. Se ha propuesto su uso como una herramienta de apoyo en el diagnóstico del asma, como indicador del control de esta enfermedad y como predictor a la respuesta a corticoides inhalados ${ }^{7}$. Esta herramienta se sustenta primariamente en las potenciales diferencias existentes entre población asmática y no asmática; además, recientemente también se han postulado diferencias según nivel de atopia. Diversos estudios han evaluado estas diferencias encontrándose, en general, un aumento en los niveles de $\mathrm{ONe}$ en suje- 
tos asmáticos, aunque también se han encontrado niveles elevados para sujetos atópicos y sobre todo sujetos asmáticos y atópicos ${ }^{8-15}$.

En este estudio se busca determinar los niveles de ONe en una población de niños en Santiago, Chile y evaluar su relación con asma y atopia. Aunque estudios de esta naturaleza se han realizado en países desarrollados, hay pocos estudios en países en desarrollo o con población latina, y a la fecha no se sabe de estudios publicados al respecto en Chile.

\section{Material y Métodos}

\section{Diseño de estudio}

Este estudio forma parte de un proyecto mayor (FONDECYT) cuyo objetivo es evaluar la relación entre la exposición a material particulado $\mathrm{MP}_{2,5}$ y salud respiratoria en niños del área norte de Santiago. El diseño es un estudio de panel, en el cual un grupo de niños asmáticos y no asmáticos fueron seguidos durante los meses de alta contaminación atmosférica (invierno) de los años 2010 y 2011, realizando mediciones repetidas de función pulmonar, ONe y síntomas. Para el presente estudio, analizamos la asociación entre $\mathrm{ONe}$ y asma, atopia y otras covariables como un diseño transversal. Para ello, las mediciones de ONe se colapsaron en una sola observación para cada niño utilizando la media. El tamaño de muestra se estimó suficiente para ver diferencias significativas entre los grupos de niños según asma y atopia, y es similar a otros estudios que han evaluado estas diferencias anteriormente.

Para cada niño se obtuvo inicialmente un cuestionario sobre antecedentes sociodemográficos y de salud, se realizó un test cutáneo de alergias y se realizaron mediciones antropométricas. El cuestionario incluyó preguntas del cuestionario ISAAC entre las cuales estaban sibilancias alguna vez, sibilancias en los últimos 12 meses, uso de inhalador preventivo y uso de inhalador de rescate. Posteriormente, se realizaron visitas periódicas en su hogar. El protocolo de estudio fue aprobado por el comité de ética de la Facultad de Medicina de la Universidad de Chile.

\section{Sujetos de estudio}

Se reclutaron 220 niños entre 6 y 14 años que vivieran y estudiaran en las comunas de Independencia y Recoleta de Santiago, Chile. Los niños asmáticos se reclutaron entre aquellos con diagnóstico médico de asma desde dos centros de salud de la zona norte de Santiago (Hospital Roberto del Río y Consultorio Cruz Melo); mientras que los niños no asmáticos se reclutaron en el consultorio mencionado y se consideró como no asmático a aquellos sin diagnóstico médico de asma (reportado por la madre y confirmado por las fichas clínicas del niño). Los niños asmáticos fueron reclutados desde algunos meses antes del estudio, al asistir a sus controles con su médico broncopulmonar, mientras los niños no asmáticos fueron reclutados mientras asistían al consultorio. A todos los participantes se les explicó los objetivos del estudio y se les solicitó la firma del consentimiento informado a los padres (o adulto responsable del niño) y del asentimiento a los niños. Se excluyó a los niños que reportaban haber fumado. Para minimizar molestias al niño y evitar sesgos por traslados, a cada niño se le visitó en su domicilio por un profesional de la salud cada dos semanas durante los 3 meses de seguimiento (alrededor de 6 mediciones por niño). Para asegurar la presencia del niño y cuidadores en el hogar y minimizar variaciones circadianas, las mediciones se realizaron entre las 16 y $20 \mathrm{~h}$.

\section{Medición de óxido nítrico exhalado (ONe) y atopia}

El ONe fue medido utilizando un equipo portátil Niox Mino, Aerocrine AB, Solna, Suecia, de acuerdo a las guías de la American Thoracic Society ${ }^{6}$. Debido a razones presupuestarias, el año 2010 solamente se pudo medir ONe en la mitad de los pacientes. De aquellos pacientes reclutados, al azar se eligieron la mitad de los asmáticos y la mitad de los no asmáticos. Para cada sujeto se realizó sólo una medición en cada visita. Para identificar la presencia de atopía, se realizó a todos los sujetos de estudio un test cutáneo de alergias según método estandarizado para los siguientes aeroalergenos: polvo habitación, mezcla de dermatophagoides, mezcla de plumas, mezcla de caspas de animales, mezcla de hongos, mezcla de polen de malezas, pastos y árboles. Se consideró reacción positiva cuando el diámetro de la pápula fue $\geq 3 \mathrm{~mm}$. Como control negativo se usó suero fisiológico y como control positivo la reacción histamina. Se definió como atópico a aquellos que presentaron al menos un resultado positivo en el test cutáneo. 


\section{Análisis estadístico}

La asociación entre variables categóricas se analizó usando test de $\chi^{2}$ con ajuste de continuidad. Para el análisis de ONe se usó el promedio de las mediciones obtenidas durante el estudio para cada sujeto. La distribución de las variables se exploró usando histogramas y gráficos QQ. Como es esperable ${ }^{7}$, ONe presentó una asimetría en la distribución de los valores por lo que los análisis se realizaron usando el logaritmo de las medias de ONe. Estadísticas sumarias se calcularon para $\mathrm{ONe}$ y para logaritmo de $\mathrm{ONe}$, y las distribuciones se graficaron usando gráficos de cajas. Para comparar las medias entre grupos se realizaron t-tests y regresiones lineales simples. Para controlar por potenciales confusores se utilizaron modelos de regresión lineales que incorporaran sexo y edad. Para los test de hipótesis se utilizó un nivel de significación de alfa de 0,05. Todos los análisis se realizaron usando el software SAS 9.3 (SAS systems, Cary, NC, EEUU).

\section{Resultados}

\section{Población en estudio}

Durante los años 2010 y 2011 se reclutaron un total de 220 niños, 114 en 2010 y 106 en 2011 (Figura 1). Previo a las mediciones se retiraron 7 pacientes. De los 111 niños seguidos el 2010, 49 niños fueron seleccionados al azar para mediciones de ONe, mientras que el 2011 todos los niños fueron evaluados. De este grupo, 143 completaron al menos 2 mediciones de ONe y el test cutáneo. Este último grupo de 143 niños se usó para los análisis.

Las características de esta muestra de niños se detallan en la Tabla 1. La media de edad fue de 9,6 años. En la muestra hubo similar número de asmáticos y no asmáticos, lo que se esperaba por diseño, número de niños y niñas y pacientes atópicos y no atópicos. Una mayor proporción de niños asmáticos presentó sibilancias (91\% vs $67 \%$ ) alguna vez o sibilancias en los últimos 12 meses ( $54 \%$ vs $22 \%$ ). De los no asmáticos, ninguno usaba inhalador preventivo ni de rescate, y de los asmáticos, alrededor de la mitad usaba inhalador preventivo y casi todos usaban de rescate. Setenta por ciento de los atópicos presentaron entre 1 y 3 reacciones positivas en el test cutáneo. Hubo una diferencia significativa en media de edad según asma (9,9 años asmáticos vs. 9,2 años para no as- máticos), pero no por atopia. No hubo diferencias significativas según sexo entre asmáticos y atópicos. Se encontró mayor porcentaje de atópicos en los asmáticos $(\mathrm{p}=0,006)$, pero no hubo diferencias en severidad según atopia. En total se realizaron 838 mediciones de $\mathrm{ONe}, 405$ en asmáticos y 433 en no asmáticos.

\section{Análisis de ONe}

Para cada niño se calculó la media de todas sus mediciones. Pese que el límite para aceptar un niño en el análisis eran 2 mediciones de ONe, $81 \%$ de los niños contó con 6 o más. Estadísticas sumarias y gráficos de caja para las medias de ONe por sujeto según nivel de asma y atopia se muestran en la Tabla 2 y Figura 2. Debido a la desviación a la derecha de las mediciones, la media geométrica tendió a ser menor que la media aritmética y los gráficos de caja se presentaron asimétricos con frecuentes valores extremos positivos. Ninguna de las comparaciones entre grupos (asmáticos vs no asmáticos, atópicos vs. no atópicos, grupos según asma y atopia) fue estadísticamente significativa.

Para evaluar el efecto de asma, atopia y la interacción entre ambos, controlando por potenciales confusores (sexo y edad), se implementaron

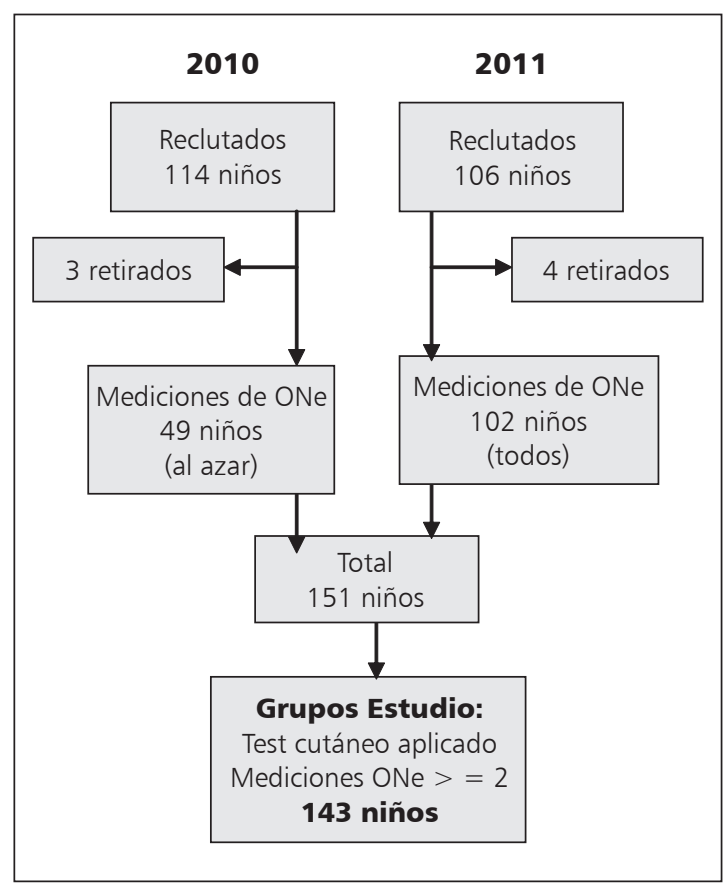

Figura 1. Diagrama de flujo de sujetos. 
modelos de regresión para el logaritmo de las medias de ONe (resultados en Tabla 3). En todos los modelos, la edad tuvo un efecto significativo (alrededor de 3\% de aumento en ONe por cada año de incremento en edad), mientras el sexo no fue significativo. Asma (modelo 1), atopia (modelo 2) y la interacción entre ambos (modelo 3 ) no mostraron un efecto significativo.

Como análisis de sensibilidad se construyeron varios modelos para determinar el efecto de distintas definiciones de asma y atopia, fiebre y efectos circadianos. Como análisis de sensibilidad para la definición de asma se usó la respuesta a la pregunta sobre sibilancias en los últimos 12 meses en vez de

Tabla 1. Características de los participantes en el estudio

\begin{tabular}{|c|c|c|c|}
\hline Características & $\begin{array}{c}\text { Todos } \\
(n=143)\end{array}$ & $\begin{array}{c}\text { No asmáticos } \\
(n=73)\end{array}$ & $\begin{array}{c}\text { Asmáticos } \\
(n=70)\end{array}$ \\
\hline Observaciones (n) & 838 & 433 & 405 \\
\hline Edad, años (media $\pm \mathrm{DE})$ & $9,6 \pm 2,2$ & $9,2 \pm 2,3$ & $9,9 \pm 2,1$ \\
\hline Sexo, M/F (\%M) & $71 / 72(50 \%)$ & $33 / 40(47 \%)$ & $37 / 32(53 \%)$ \\
\hline Atopia, (\%) & $72(50 \%)$ & $28(39 \%)$ & $44(61 \%)^{\dagger}$ \\
\hline $\begin{array}{l}\text { Número de reacciones (\%) } \\
\begin{array}{l}0 \\
1-3 \\
>3\end{array}\end{array}$ & $\begin{array}{l}71(50 \%) \\
50(35 \%) \\
22(15 \%)\end{array}$ & $\begin{array}{c}45(62 \%) \\
20(28 \%) \\
8(11 \%)\end{array}$ & $\begin{array}{l}26(37 \%)^{\dagger} \\
30(46 \%)^{\dagger} \\
14(20 \%)^{\dagger}\end{array}$ \\
\hline Peso $(k g)$ & $41,0 \pm 13,6$ & $38,0 \pm 12,7$ & $44,0 \pm 13,9^{*}$ \\
\hline Talla (cm) & $138,4 \pm 13,7$ & $135,7 \pm 13,9$ & $141,3 \pm 13,0$ * \\
\hline Circunferencia abdominal (cm) & $74,8 \pm 12,1$ & $73,1 \pm 12,8$ & $76,5 \pm 11,5$ \\
\hline $\mathrm{BMI}\left(\mathrm{Kg} / \mathrm{m}^{2}\right)$ & $20,8 \pm 3,9$ & $20,1 \pm 3,8$ & $21,5 \pm 4,0$ \\
\hline Sibilancias alguna vez (\%) & $113(79 \%)$ & $49(67 \%)$ & $64(91 \%)^{\dagger}$ \\
\hline Sibilancias 12 meses (\%) & $54(38 \%)$ & $16(22 \%)$ & $38(54 \%)^{\dagger}$ \\
\hline Inhalador de rescate & $67(47 \%)$ & $0(0 \%)$ & $67(96 \%)^{\dagger}$ \\
\hline Inhalador preventivo & $40(28 \%)$ & $0(0 \%)$ & $40(57 \%)^{\dagger}$ \\
\hline
\end{tabular}

†Valor $\mathrm{p}<0,05$ para test $\chi^{2}$ asmáticos vs no asmáticos. *Valor $\mathrm{p}<0,05$ para test $\mathrm{t}$ asmáticos vs no asmáticos.

Tabla 2. Distribución de ONe, por nivel de asma y atopia

\begin{tabular}{|lccccccc|}
\hline Tipo & $\mathbf{n}$ & Media $\pm \mathbf{D E}$ & Min. & Max. & Media Geom. & Límites 95\% & Valor $\mathbf{p}^{*}$ \\
$\begin{array}{l}\text { Asma } \\
\quad \text { Sí }\end{array}$ & 70 & $17,5 \pm 11,5$ & 6,7 & 67,8 & 14,3 & $5,3-39,0$ & $0,19^{\dagger}$ \\
$\quad$ No & 73 & $14,7 \pm 6,2$ & 6,3 & 37,8 & 12,9 & $6,1-27,7$ & \\
Atopia & & & & & & & \\
$\quad$ Sí & 73 & $17,6 \pm 11,7$ & 6,7 & 67,8 & 14,2 & $4,9-41$ & $0,25^{\dagger}$ \\
$\quad$ No & 70 & $14,5 \pm 5,3$ & 6,3 & 37,8 & 13,0 & $6,7-25,2$ & \\
Asma y atopia & & & & & & & \\
$\quad$ Asma-atopia & 45 & $19,5 \pm 13,6$ & 6,7 & 67,8 & 15,3 & $4,9-48,0$ & \\
$\quad$ Asma-no atopia & 25 & $14,0 \pm 4,8$ & 8,3 & 27,7 & 12,7 & $6,7-23,9$ & \\
$\quad$ No asma-no atopia & 28 & $14,6 \pm 7,1$ & 6,7 & 33,8 & 12,5 & $5,2-30,2$ & \\
$\quad$ No asma-atopia & 45 & $14,8 \pm 5,6$ & 6,3 & 37,8 & 13,2 & $6,7-26,1$ & \\
\hline
\end{tabular}

En negrita, comparaciones significativas alfa 0,05. ${ }^{*}$ Tests para log $(\mathrm{ONe}) .{ }^{\dagger} \mathrm{t}$-test. ${ }^{\mathrm{F}} \mathrm{F}$-test. 


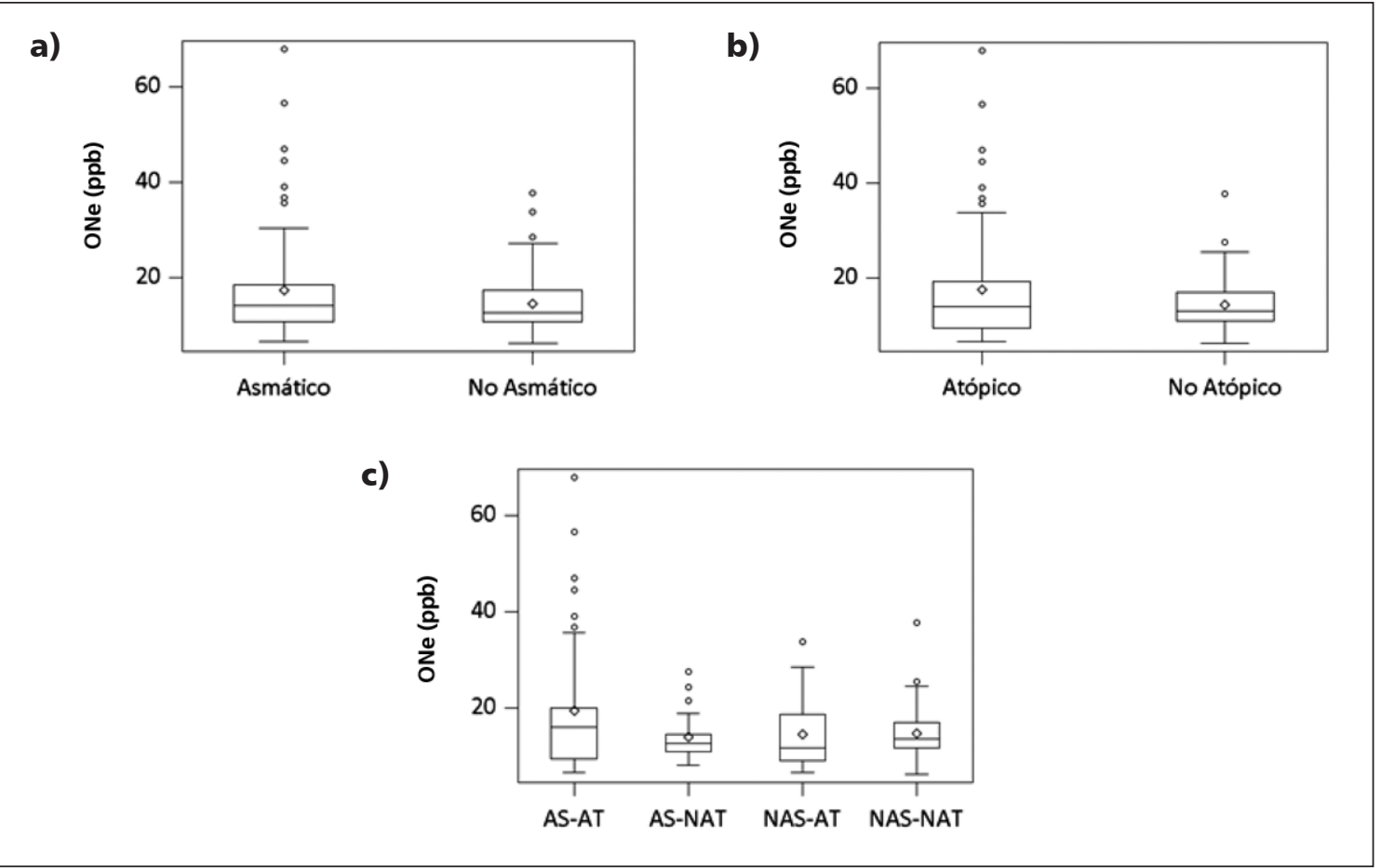

Figura 2. Distribución de medias por individuo de ONe por categorías de asma y atopia. a) según asma; b) según atopia y c) según asma y atopia.

Tabla 3. Modelos de regresión para log de las medias de ONe

\begin{tabular}{|c|c|c|c|c|c|}
\hline Modelo & Estimador & Error estándar & Exp (est) & Límites 95\% & Valor $\mathbf{p}^{*}$ \\
\hline \multicolumn{6}{|l|}{ Modelo 1: Asma } \\
\hline Intercepto & 1,961 & 0,192 & 7,1 & $4,9-10,4$ & $<0,001$ \\
\hline Sexo (Masculino) & 0,048 & 0,073 & 1,049 & $0,91-1,211$ & 0,51 \\
\hline Edad (1 año) & 0,063 & 0,017 & 1,065 & $1,032-1,1$ & $<0,001$ \\
\hline Asma (Asmático) & 0,057 & 0,074 & 1,059 & $0,916-1,223$ & 0,44 \\
\hline \multicolumn{6}{|l|}{ Modelo 2: Atopia } \\
\hline Intercepto & 1,898 & 0,186 & 6,7 & $4,6-9,6$ & $<0,001$ \\
\hline Sexo (Masculino) & 0,038 & 0,073 & 1,039 & $0,901-1,197$ & 0,60 \\
\hline Edad (1 año) & 0,065 & 0,016 & 1,067 & $1,034-1,102$ & $<0,001$ \\
\hline Atopia (Atópico) & 0,068 & 0,072 & 1,071 & $0,929-1,234$ & 0,35 \\
\hline \multicolumn{6}{|c|}{ Modelo 3: Asma y atopia } \\
\hline Intercepto & 1,926 & 0,187 & 6,9 & $4,8-9,9$ & $<0,001$ \\
\hline Sexo (Masculino) & 0,058 & 0,074 & 1,059 & $0,917-1,224$ & 0,44 \\
\hline Edad (1 año) & 0,062 & 0,016 & 1,064 & $1,03-1,099$ & $<0,001$ \\
\hline Asma* Atopia & & & & & $0,31^{\dagger}$ \\
\hline - Asma-atopia & 0,101 & 0,092 & 1,106 & $0,924-1,324$ & 0,27 \\
\hline - Asma-no atopia & $-0,076$ & 0,108 & 0,927 & $0,751-1,145$ & 0,48 \\
\hline - No asma-no atopia & $-0,056$ & 0,105 & 0,946 & $0,77-1,161$ & 0,60 \\
\hline
\end{tabular}

En negrita, comparaciones significativas alfa 0,05. ${ }^{*}$ Tests para log $(\mathrm{ONe}) .{ }^{\dagger} \mathrm{t}$-test. ${ }^{\mathrm{F}} \mathrm{F}$-test. 
la etiqueta diagnóstica. El análisis no mostró asociación entre esta variable y $\mathrm{ONe}$. Con respecto a atopia, se realizó un análisis utilizando como variables predictoras el número de reacciones positivas en el test cutáneo (testeado tanto como variable continua como variables categóricas agrupadas en 0 reacciones, 1 a 3 reacciones y más de 3 reacciones). Ninguna de estas variables fue significativa. Para ver el impacto de fiebre y ciclos circadianos se construyeron modelos de mediciones individuales con interceptos azarosos. La variable fiebre/resfrío durante la medición presentó un impacto significativo en $\mathrm{ONe}$ (aumentándolo); sin embargo, los efectos de asma y atopia no fueron diferentes a los modelos informados anteriormente. Con respecto a los modelos circadianos, se incorporó la variable hora de medición a los modelos. Esta variable no fue significativa y no cambió los resultados para asma y atopia.

Se ha sugerido en literatura usar un punto de corte de $25 \mathrm{ppb}$ como para diagnosticar asma. No se encontró una asociación significativa $(\mathrm{p}=0,24)$ entre los niños con valor sobre $25 \mathrm{ppb}$ y asma. Sí se encontró una asociación significa con atopia $(\mathrm{p}=0,04), y$ con los niños asmáticos atópicos comparados con el resto $(\mathrm{p}=0,03)$.

\section{Discusión}

En nuestro estudio evaluamos los niveles de ONe en un grupo de niños en Santiago de Chile según asma y atopia. No encontramos diferencias estadísticamente significativas según nivel de asma o atopia. Estos resultados fueron robustos a cambio de definición de asma por presencia de sibilancias en los últimos 12 meses, definición de atopia, ciclos circadianos y síntomas de infección respiratoria durante las mediciones.

Varios autores han determinado el impacto de asma y atopia en los niveles de $\mathrm{ONe}^{8-15}$. Tanto asma como atopia por separado presentaron en general aumentos en ONe mayores a 20\%, llegando incluso a triplicar los valores en algunas ocasiones. Además, se reportan niveles significativamente más altos de ONe para niños asmáticos y atópicos en conjunto, con respecto a lo esperado por el impacto del asma y atopia por separado. Estas diferencias se han visto incluso en población de niños bajo tratamiento con corticoides ${ }^{9-11}$. No son claras las razones para no haber observado diferencias en niveles de ONe según asma y atopia en nuestro estudio. Dado el tamaño de muestra se cuenta con el poder suficiente para ver diferencias del orden de magnitud de las observadas en la literatura (lo que se aprecia en lo estrecho de los intervalos de confianza y errores estándar de los estimados de asociación. Probables razones pueden deberse a i) bajos niveles observados en aquellos individuos en los que se esperaban valores altos (asmáticos, atópicos); y ii) altos niveles observados para aquellos individuos en los que se esperaban valores bajos (no asmáticos, no atópicos).

Con respecto a la primera posibilidad, nuestros pacientes asmáticos fueron reclutados desde una unidad de referencia especializada, la mayoría en tratamiento con corticoides inhalados probablemente en dosis suficiente para el control de la enfermedad lo que puede incidir en observar valores menores a lo esperado para asmáticos. Sin embargo, otros estudios han observado diferencias incluso en población tratada con corticoides pero no se sabe el grado de control de la enfermedad en esos estudios. En Chile el tratamiento del asma está garantizado por el Estado, por lo que especulamos sobre la mejor cobertura al tratamiento comparado con otros estudios. Una segunda alternativa que especulamos puede deberse a que la población de asmáticos estudiados en sí (por factores genéticos, ambientales tales como nutrición, otros) presenta niveles menores de ONe comparado a otras poblaciones. Otra posibilidad es que estudios internacionales han sobreestimado el impacto debido a problemas de diseño o sesgos de selección, por ejemplo selección de individuos asmáticos con mal control del asma. La falta de asociación de los niveles de $\mathrm{ONe}$ con atopia puede explicarse por las características de la población estudiada; en que los pacientes presentan un alto porcentaje de atopia comparado con otras poblaciones, pero que a la vez no están expuestos alergenos (niños atópicos a caspa de gato, pero que no posean el animal) o estos alérgenos bajo las condiciones de exposición o no son capaces de gatillar una reacción de inflamatoria de la vía aérea. La falta de efecto de asma no puede ser explicada por talla o peso dado que los asmáticos en general tenían más peso y talla que los no asmáticos, por lo que controlar por esta variable potencialmente podría disminuir el efecto de asma.

Con respecto a la segunda alternativa de explicación, niveles altos para niños no asmáticos 
y no atópicos, se destaca que en nuestro estudio los niveles de $\mathrm{ONe}$ en población no asmática y no atópica son más elevados que los valores promedios publicados ${ }^{16}$. Por ejemplo para un niño de 6 años, los niveles esperados según Buchvald son 8,2 ppb, mientras lo esperado según modelo 3 en Tabla 3 de nuestro estudio es de 10,0 ppb. Esto podría explicarse porque nuestro estudio se realizó en los meses fríos del año, coincidiendo con niveles elevados de contaminación, alta circulación de virus respiratorios y condiciones de baja ventilación en hogares favoreciendo la acumulación de contaminantes intradomiciliarios. Se están preparando trabajos analizando el impacto de estas variables en salud respiratoria de esta cohorte incluyendo el efecto en ONe. Una segunda alternativa es que la población estudiada en sí tenga valores más altos comparados con otras poblaciones.

Reconocemos como posible debilidad del estudio errores en la clasificación de los pacientes que podría explicar estos resultados negativos. Con respecto a clasificación como asmático, los casos se obtuvieron a través de fichas que incluye diagnóstico de asma por un médico broncopulmonar, por lo que es poco probable esta desclasificación. Sin embargo, los controles pueden ser asmáticos no diagnosticados, que incluso pueden estar en tratamiento sin haber sido declarados como tales o sin el conocimiento de los familiares.

Las fortalezas de este estudio radican en que las mediciones se realizaron en un ambiente real, en el hogar del paciente, con mediciones seriadas y por personal adecuadamente entrenado. No se seleccionaron pacientes asmáticos o controles según gravedad de la enfermedad o eventos puntuales, ni las mediciones fueron hechas en función del estado del paciente.

En conclusión en nuestro estudio no encontramos diferencias significativas en los niveles de ONe según niveles de asma y atopia.

Agradecimientos: Hospital Roberto del Río y Consultorio Cruz Melo por ayuda en reclutamiento de los pacientes. Este estudio fue financiado por proyecto FONDECYT 11090309.

\section{Referencias}

1. Bousquet J, Jeffery PK, Busse WW, Johnson M, Vignola AM. Asthma. From bronchoconstriction to airways inflammation and remodeling. Am J Respir Crit Care
Med 2000; 161 (5): 1720-45.

2. Barbato A, Turato G, Baraldo S, Bazzan E, Calabrese F, Tura $\mathrm{M}$, et al. Airway inflammation in childhood asthma. Am J Respir Crit Care Med 2003; 168 (7): 798-803.

3. Jatakanon A, Lim S, Kharitonov SA, Chung KF, Barnes PJ. Correlation between exhaled nitric oxide, sputum eosinophils, and methacholine responsiveness in patients with mild asthma. Thorax 1998; 53 (2): 91-5.

4. Berlyne GS, Parameswaran K, Kamada D, Efthimiadis A, Hargreave FE. A comparison of exhaled nitric oxide and induced sputum as markers of airway inflammation. J Allergy Clin Immunol 2000; 106 (4): 638-44.

5. Warke TJ, Fitch PS, Brown V, Taylor R, Lyons JD, Ennis $\mathrm{M}$, et al. Exhaled nitric oxide correlates with airway eosinophils in childhood asthma. Thorax 2002; 57 (5): 383-7.

6. ATS/ERS recommendations for standardized procedures for the online and offline measurement of exhaled lower respiratory nitric oxide and nasal nitric oxide, 2005. Am J Respir Crit Care Med 2005; 171 (8): 912-30.

7. Dweik RA, Boggs PB, Erzurum SC, Irvin CG, Leigh MW, Lundberg JO, et al. An official ATS clinical practice guideline: interpretation of exhaled nitric oxide levels (FONE) for clinical applications. Am J Respir Crit Care Med 184 (5): 602-15.

8. Brussee JE, Smit HA, Kerkhof M, Koopman LP, Wijga $\mathrm{AH}$, Postma DS, et al. Exhaled nitric oxide in 4-year-old children: relationship with asthma and atopy. Eur Respir J 2005; 25 (3): 455-61.

9. Hervas D, Milan JM, Garde J. Differences in exhaled nitric oxide in atopic children. Allergol Immunopathol (Madr) 2008; 36 (6): 331-5.

10. Yao TC, Ou LS, Lee WI, Yeh KW, Chen LC, Huang JL, et al. Exhaled nitric oxide discriminates children with and without allergic sensitization in a population-based study. Clinical and Experimental Allergy 2011; 41 (4): 556-64.

11. Crane J, Lampshire P, Wickens K, Epton M, Siebers R, Ingham $\mathrm{T}$, et al. Asthma, atopy and exhaled nitric oxide in a cohort of 6-yr-old New Zealand children. Pediatric Allergy and Immunology 2012; 23 (1): 59-64.

12. Barreto M, Villa MP, Monti F, Bohmerova Z, Martella S, Montesano M, et al. Additive effect of eosinophilia and atopy on exhaled nitric oxide levels in children with or without a history of respiratory symptoms. Pediatric Allergy and Immunology 2005; 16 (1): 52-8.

13. Cibella F, Cuttitta G, La Grutta S, Passalacqua G, Viegi G. Factors that influence exhaled nitric oxide in Italian schoolchildren. Annals of Allergy Asthma \& Immuno$\operatorname{logy} 2008 ; 101$ (4): 407-12. 
14. Silvestri M, Sabatini F, Spallarossa D, Fregonese L, Battistini E, Biraghi MG, et al. Exhaled nitric oxide levels in non-allergic and allergic mono- or polysensitised children with asthma. Thorax 2001; 56 (11): 857-62.

15. Jouaville LF, Annesi-Maesano I, Nguyen LT, Bocage AS, Bedu M, Caillaud D. Interrelationships among asthma, atopy, rhinitis and exhaled nitric oxide in a populationbased sample of children. Clin Exp Allergy 2003; 33 (11): 1506-11.

16. Buchvald F, Baraldi E, Carraro S, Gastón B, De Jongste J, Pijnenburg MW, et al. Measurements of exhaled nitric oxide in healthy subjects age 4 to 17 years. J Allergy Clin Immunol 2005; 115 (6): 1130-6. 